\title{
Información fraudulenta sobre salud en internet
}

\author{
Por Belén Castelló-Zamora
}

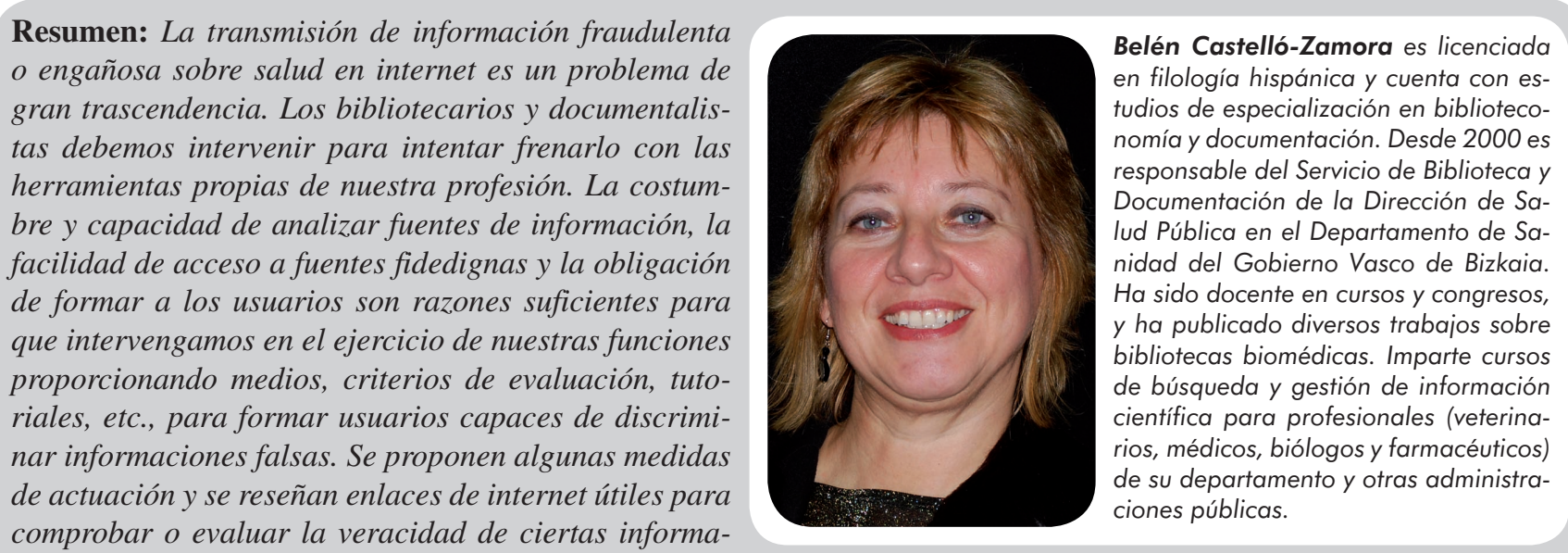
ciones.

Palabras clave: Información sobre salud, Internet, Fraudes, Leyendas urbanas, Bulos, Cadenas de e-mail.

\section{Title: Fraudulent information about health on the internet}

Abstract: The transmission of fraudulent or misleading information on health through the internet is a major problem. Librarians must intervene to curb the scourge, using the tools of our profession: the tendency and ability to analyze sources of information, easy access to reliable sources and the obligation to train users. These resources are reasons enough for us to intervene as part of our duty to provide media, evaluation criteria, tutorials, etc. that develop users'capacity to recognize false information. Some performance measures are proposed and useful web links to verify or evaluate the veracity of certain information are suggested.

Keywords: Health information, Internet, Frauds, Urban legends, Hoax, E-mail chain letters.

Castelló-Zamora, Belén. "Información fraudulenta sobre salud en internet”. El profesional de la información, 2010, mayo-junio, v. 19, n. 3, pp. 292-295.

DOI: 10.3145/epi.2010.may.10

\section{LA INFORMACIÓN SOBRE} SALUD es un terreno propicio para engaños ya que es un tema sensible, que nos interesa $y$ afecta. Las implicaciones que tiene el mantenimiento de nuestra salud y de las personas que nos rodean, nos hacen vulnerables y víctimas potenciales del engaño.

En internet abundan las páginas fraudulentas animadas por diversas razones, desde afán de protagonismo y "ocio" mal entendido (bromas pesadas, hoax), a incluso intenciones de causar daño a terceros.

Como profesionales de la información pasan ante nuestros ojos multitud de fuentes en internet de dudosa o nula veracidad. Lo mismo puede decirse sobre algunas cadenas de correo electrónico, que constituyen fuentes de desinformación que tienen por objeto no sólo vender, sino "alertar", "aconsejar" o calumniar a la competencia.

Ante esta situación, el usuario medio no maneja las herramientas que son habituales en el entorno de los profesionales de la información

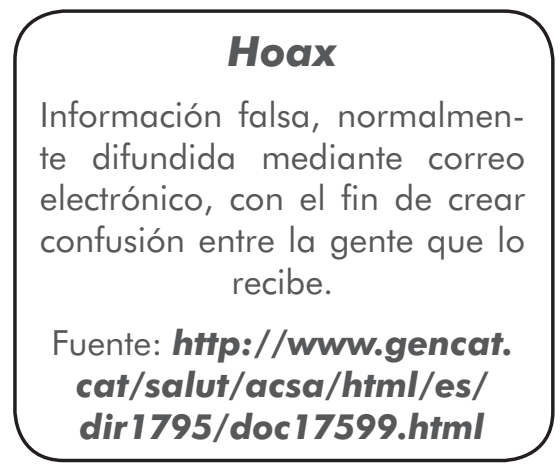

sobre salud. Generalmente no ha oído hablar de términos especializados como sellos de calidad, el código $H O N$, o los programas $W M A$ o WMC. Ni siquiera suele fijarse en los datos que proporciona una web transparente al usuario avisado: mención de responsabilidad, fuentes, fechas, interpretación de los urls, enlaces ofrecidos y otras cuestiones que los especialistas tienen en cuenta para tratar de discernir si una información inicialmente dudosa es fidedigna o no.

Como profesionales de la información tenemos una enorme labor que realizar para educar a la gente también en este aspecto. Tratándose de información biomédica y de salud en general, el asunto es más inexcusable si cabe. La estafa, la 
cura milagrosa o la intoxicación informativa en un tema tan compro- metido tiene trascendencia en los ciudadanos y necesita de una pon- deración con espíritu científico y una buena dosis de sentido común.

\section{HONcode, WMA y WMC}

El Código de conducta (HON code) de la fundación Health on the Net para sitios web médicos y de salud se dirige a uno de los principales temas sobre atención sanitaria en internet: la fiabilidad y la credibilidad de la información. HONcode no es un sistema para premiar ni tiene la intención de evaluar la calidad de la información proporcionada en una web. Sólo define un grupo de reglas para:

- comprometer a los creadores de webs con normas éticas básicas en la presentación de la información;

- ayudar a los usuarios a conocer siempre la fuente y el objetivo de los datos leídos.

Web Mèdica Acreditada (WMA) es un programa de calidad para sitios web con información médica. Certifica mediante un proceso de revisión que las webs que se adhieren voluntariamente a dicho programa, cumplen un conjunto de requisitos de calidad, creando una comunidad virtual de confianza en internet para usuarios y profesionales de salud.

El proyecto Webs Médicas de Calidad (WMC) es una iniciativa en internet para la mejora de la calidad de webs de contenido sanitario en lengua española. Su sello de calidad avala a sitios web que han superado un riguroso proceso de acreditación, y a los que se les realiza un seguimiento periódico, con el fin de velar que sigan cumpliendo un código de conducta y unos criterios de calidad.

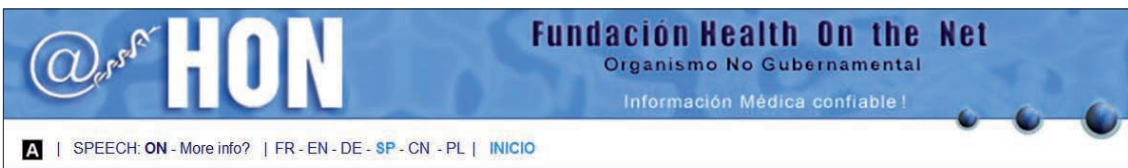

A | SPEECH: ON - More into? | FR - EN - DE - SP - CN - PL | INICIO

PACIENTE I PERSONA PROFESIONAL MÉDICO

Los servicios ofrecidos por HON
HONcode

Certificación Honcode: Mejora la calidad de la
información médica en información médic

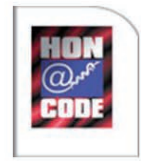

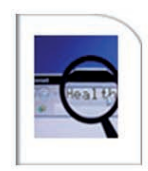

HONsearch

Büsqueda de sitios Web Büsqueda de sitios Web
confiables que se ajustan
a HONcode

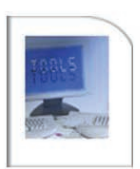

HONtools

Utilice nuetros servicios y ameliorar su
sution

Figura 1. HONcode, http://www.hon.ch

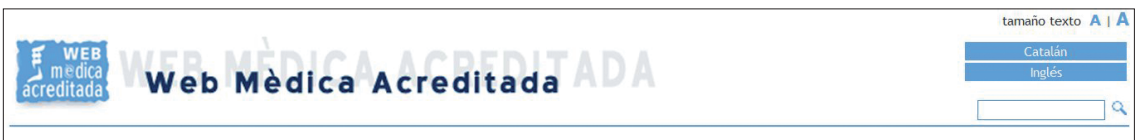

\begin{tabular}{|l|}
\hline Web Mèdica Acreditada \\
\hline Presentación \\
\hline Código de Conducta \\
\hline Solicitud \\
\hline Indice de Webs \\
Médicas Acreditadas \\
Colaboraciones \\
Contactar \\
\hline Información de Interés \\
\hline Noticias \\
\hline Publicaciones WMA \\
\hline Congresos \\
\hline Aspectos Legales \\
\hline Web Plus \\
\hline Usuarios de Internet \\
\hline
\end{tabular}

Web Mèdica Acreditada (WMA) es un programa de calidad dirigido a páginas web con información médica, que certifica mediante un proceso de revisión, que las webs que se adhieren voluntariamente a dicho programa cumplen un conjunto de requisitos de calidad, creando una comunidad virtual de confianza en Internet tanto para los usuarios en general como para los profesionales de salud. Más información

\begin{tabular}{|l||l|l|l|l}
\hline Noticias & Publicaciones \\
\hline
\end{tabular}

" eHealth week 2010. Barcelona: Durant los días 15 a 18 de marzo se celebrará en Barcelona la Conferencia Ministerial de Alto Nivel sobre eHealth y las Tecnologias de la Información Sanitarias. Uso de las TIC en Atenció Primaria (en catalán): EI Fòrum Català de Informació i Salut (CIS) y TIC Salut en colaboracion con diversas entidades organizan un debate virtual sobre aspectes relacionados con el impacto de las TiC en la Atención Primaria.

Retos éticos de la e-salud: Publicado el informe num. 20 de los "Cuadernos de la Fundacio Grifols"

¿Qué es Web Mèdica colaboración con el Observatorio de la

" Mayer MA, Leis A. Conceptos y aplicaciones de la Web 3.0: una introducción para médicos. Aten Primaria. 2010 [Epub ahead of print].

Mayer MA, Leis A, Sanz F. La información de salud en Internet y los sellos de confianza como Aten Primaria. 2009;41(10):534-44.

" Lupiáñez F, Mayer MA, Torrent J. Opportunities and challenges of web 2.0 within the health care systems: an empirical exploration. Informatics for

Figura 2. Web Mèdica Acreditada, http://wma.comb.es

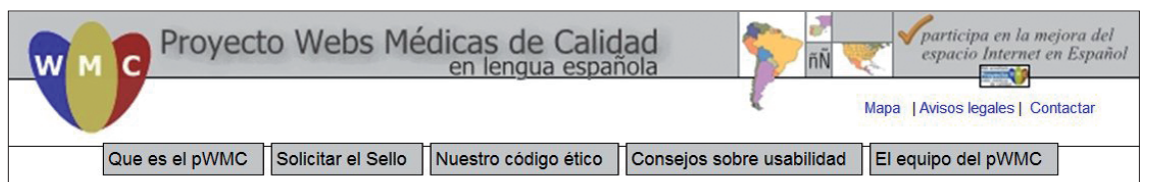

\section{Sello de calidad del pWMC}

El proyecto Webs Médicas de Calidad es una iniciativa en Internet, que tiene como objetivo la mejora de la calidad de las Webs de contenido sanitario relativo a la salud humana, desarrolladas en lengua española.

Su sello de calidad avala a sitios web que han superado un riguroso proceso de acreditación, y a los que se les realiza un seguimiento periódico, con el fin de velar que siguen cumpliendo nuestro código de conducta y los criterios de calidad que identifican nuestro sello.

Este procedimiento de acreditación es completamente gratuito y voluntario. saber más ....

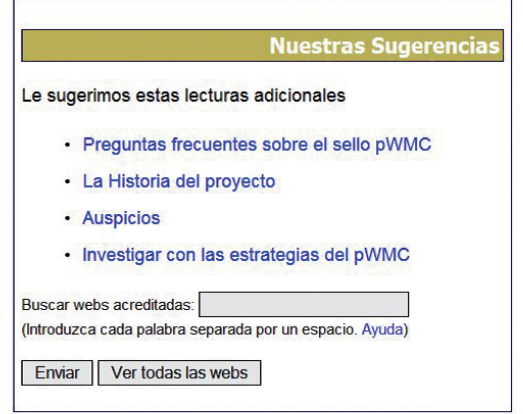

\begin{tabular}{|l|l|}
\hline Enviar & Ver todas las webs \\
\hline
\end{tabular}

Figura 3. Webs Médicas de Calidad, http://www.pwmc.org 


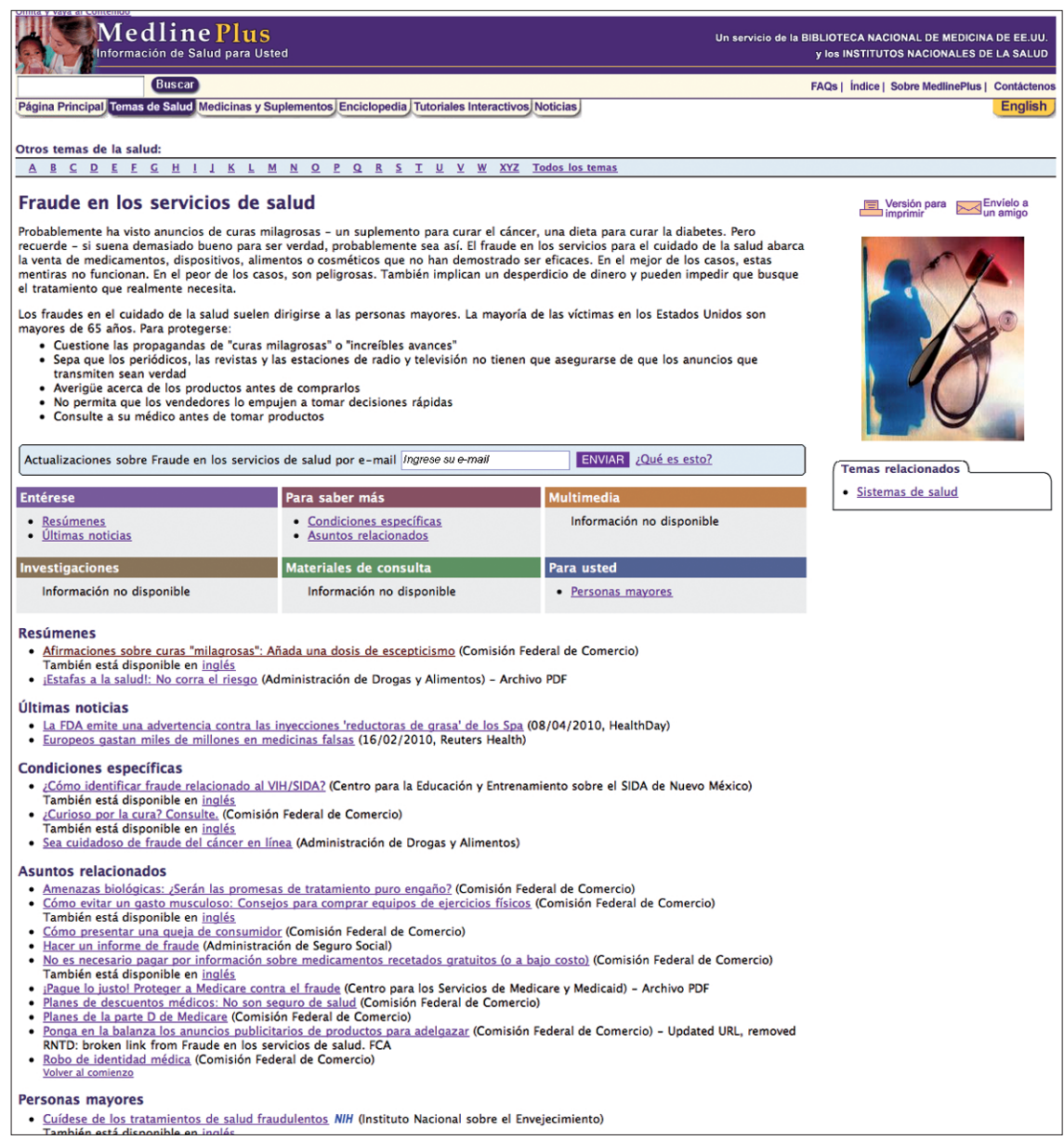

Figura 1. Información sobre fraudes de Medline Plus http://www.nlm.nih.gov/medlineplus/spanish/healthfraud.html

El concepto de alfin (alfabetización informacional) encaja perfectamente en la destreza de saber discriminar una información fraudulenta. Podemos actuar de varias maneras. Por ejemplo, dentro de la formación continuada de nuestros usuarios, no sólo ayudándoles a conocer las fuentes fiables y reconocer las falsas, sino también inculcando un sano escepticismo para juzgar los resultados de sus búsquedas y las informaciones que reciben en forma de cadenas de e-mail. La educación en información se convierte en educación en salud.

Las webs de nuestras bibliotecas y centros de documentación (o de las organizaciones en las que estamos integrados) son lugares perfectos para ayudar al profesional de la salud y al ciudadano en general a familiarizarse con sellos de calidad, análisis de webs y formas de testarlas, proporcionándoles sec- ciones con enlaces recomendados sobre informaciones de este tipo en internet o tutoriales de elaboración propia, realizados por expertos.

\section{"El concepto de alfabetización informacional encaja en la destreza de saber discriminar una información fraudulenta"}

En cuanto a las cadenas de email sobre salud y enfermedad que tanto abundan, debemos tener una intervención proactiva y responsable, ayudando a desenmascarar las falsas informaciones que se distribuyen incluso entre profesionales de ciencias de la salud, y que a veces ellos mismos reenvían a colegas sin comprobar ni evaluar.
Todos hemos recibido y leído correos electrónicos sobre cáncer, virus, niños necesitados de transfusión, contagios inverosímiles, supervivencias a ataques cardiacos..., y nos corresponde detener la transmisión o mejor aún, enviar al remitente la información que manejamos para que pueda contrastarla y decidir.

Existen muchas iniciativas que tratan de neutralizar esta lacra. Curiosamente están tuteladas casi siempre por informáticos (circunstancia ésta que nos debería hacer reflexionar), y no por gestores de información, bibliotecarios y documentalistas. También algunas organizaciones de la salud tienen un espacio dedicado a las leyendas urbanas y otras falacias y suelen ser citados por los anteriores. Tal vez en las secciones de La biblioteca responde se haya contestado más de una vez a estas cuestiones, pero esto suele producirse a demanda de un usuario.

A veces nos podríamos preguntar: ¿quien vigila al vigilante? ¿será verdad que es mentira? Pero estas webs "rompecadenas" nos ofrecen fiabilidad y transparencia, pues aportan datos, fuentes, bibliografía, enlaces que sí existen.

A continuación se incluyen algunas de estas webs que cumplen requisitos similares. Generalmente analizan la estructura sintáctica y semántica, que es recurrente en las cadenas de correo: anonimia, lenguaje exaltado y alarmista, referencias a grandes compañías comerciales (¿la teoría de la conspiración?), amenaza subliminal de grandes desgracias en caso de no reenviarlas, ausencia de fuentes científicas donde comprobar nada. A veces citan fuentes inexistentes.

Casi todas las webs consultadas y que se citan a continuación presentan una breve ficha donde se data el origen de la cadena, se describe sucintamente la materia y se indica si la información es falsa, 


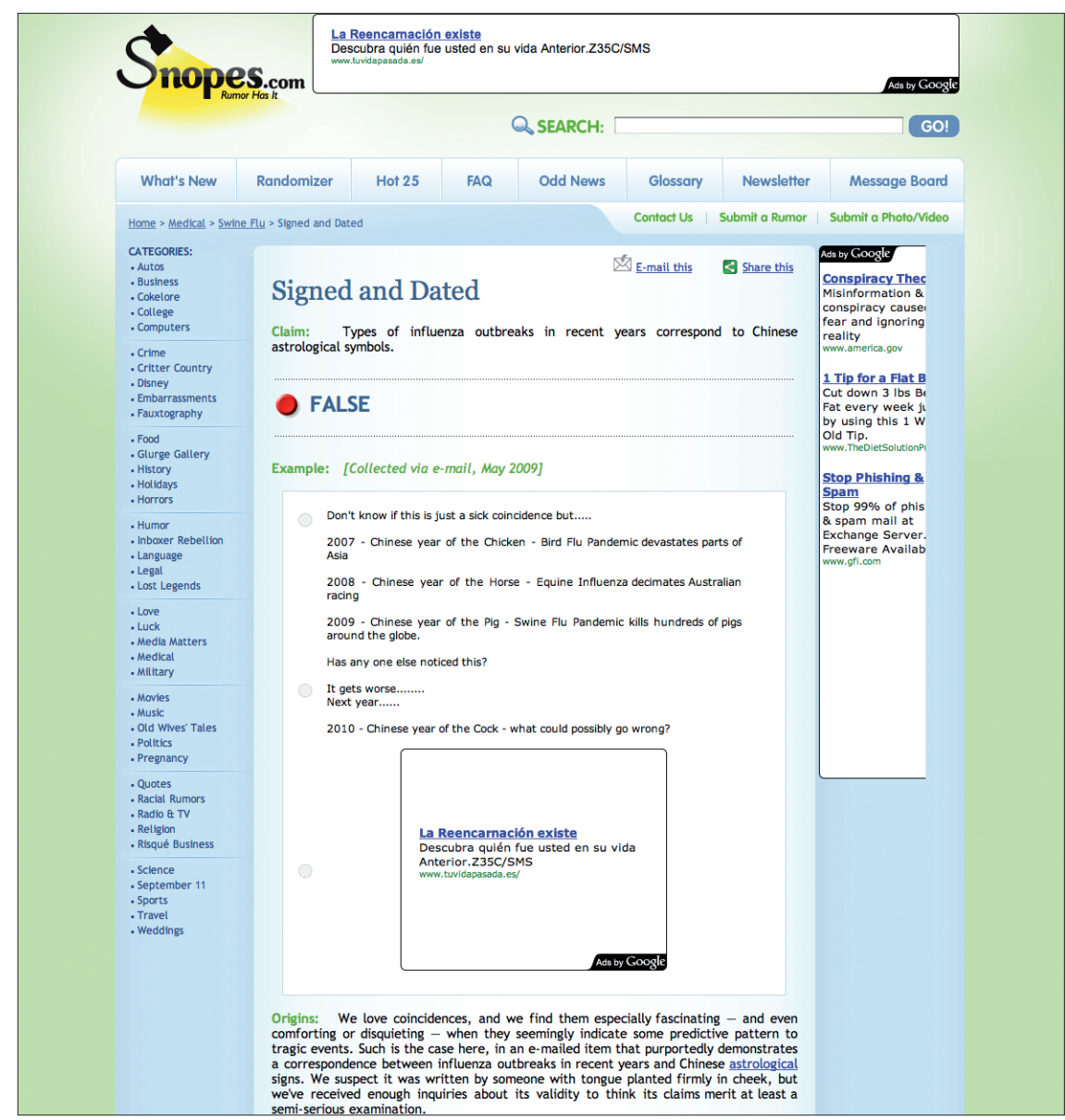

Figura 2. Relación entre la astrología china y epidemias, en Snopes

verdadera o puede tener una parte de cada. Algunas rastrean el origen geográfico, idioma original, etc.

Todas aportan bibliografía o referencias a webs de organizaciones reconocidas relacionadas con la salud.

\section{"Nos corresponde detener la transmisión o mejor aún, enviar al remitente la información que manejamos para que pueda contrastarla y decidir"}

\section{Webs generales}

En general todas ellas reproducen y analizan el estilo de la cadena, siempre remiten a instituciones y organismos oficiales relacionados con la salud o con otras webs dedicadas a descubrir falacias. / Medical.

http://urbanlegends.about.com/od/ medical/Health_Medical.htm

Breakthechain

http://www.breakthechain.org/

Rompecadenas

http://www.rompecadenas.com.ar/ cpr.htm

Snopes. Rumor has it. Medical medical.asp

Vsantivirus: estar informado para estar seguro

http://www.vsantivirus.com/hoaxes. htm

\section{Sitios de organismos oficiales}

ACSA. Hoax.

http://www.gencat.cat/salut/acsa/

Información sobre hoax de ACSA, la Agencia Catalana de Seguridad Alimentaria
About.com: Urban legends. Health http://www.snopes.com/medical/ html/es/dir1795/doc17599.html
CDC Centers for Disease Control and Prevention. Health related hoaxes and rumors.

Tiene una sección dedicada a leyendas urbanas y falsos rumores sobre salud. Muchas de las páginas "rompecadenas" remiten a los $C D C$.

http://www.cdc.gov/hoaxes_rumors. html

http://www.cdc.gov/ncidod/klinger man_hoax.htm

http://www.cdc.gov/hiv/resources/ qa/hoaxl.htm

FTC. Información de la FTC para consumidores http://www.ftc.gov/bcp/edu/pubs/ consumer/health/shea07.shtm

Es una publicación producida por la Federal Trade Commission (FTC) de Estados Unidos en cooperación con la Food and Drug $A d$ ministration (FDA). Enseña cómo distinguir sitios web que ofrecen a la venta productos milagrosos.

\section{MedlinePlus. Health Fraud.} http://www.nlm.nih.gov/medlineplus/ healthfraud.html

Muy indicado para los ciudadanos en general, educativo y sencillo. Sugiere enlaces a otras organizaciones.

MedlinePlus. Fraude en los servicios de salud.

http://www.nlm.nih.gov/medlineplus/ spanish/healthfraud.html

El anterior en castellano, muy educativo y sencillo, pero con menos material que la versión en inglés.

Quackwatch: your guide to quackery, health fraud, and intelligent decisions.

http://www.quackwatch.org/

Belén Castelló-Zamora. Gobierno Vasco, Departamento de Sanidad y Consumo, Centro de Documentación de Sanidad.

bibliobi-san@ej-gv.es 